\title{
Aménagement hydroélectrique de l'Oued Djendjen supérieur
}

\author{
Cheminée d'équilibre et conduite forcée de l'usine souterraine de Mansouria
}

Adaptation des ouvrages à la nature des terrains rencontrés

\section{The upper Wadi Djendjen hydro-electric development}

\author{
Surge tank and penstock for the Mansouria underground powerhouse \\ Adaptation of the structures to geological formations traversed \\ PAR E. MÉNÉROUD

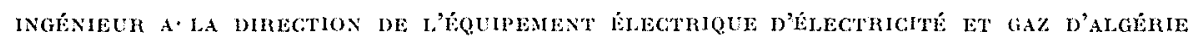

Communication présentée à la Société Hydrotechnique de France

(Paris, 14 mars 1958)

\begin{abstract}
Projet de cheminée déversante entierement souterraine à deux chambres (1 inférieure, 1 supérieure), constituées par des galeries et reliées par un puits. Ce projet s'est révélé plus économique que le type classique que la nature du terrain rendait trop couteux.

Conduite force : galerie souterraine de pente 1/1 excavée dans le calcaire du lias, suivie à sa partie inférieure d'un palies dans la dolomie; blindage mince, la majeure partie des contraintes étant supportée par le massif rocheux. La présence des fissures et des diaclases bourrées d'argile par endroits a incité à la prisdence et des essais de prérevêtement précontraint ont été réalisés dans les dolomies et dans les calcaires.
\end{abstract}

L'aménagement hydroélectrique de l'Oued Djendjen Supérieur est situé en Petite Kabylie dans le massif du Babor (2 $004 \mathrm{~m}$ ), près du village de Ziama Mansouria.

Cet aménagement consiste en la mise en valeur d'un ensemble de circonstances naturelles favorables qu'il est relativement rare de voir se présenter simultanément dans des conditions aussi propices; l'essentiel tient dans l'énoncé

\begin{abstract}
Design for a completely underground, double chamber overflowing surge tank, upper and lower chambers consisting of tunnels connected by a shaft. This design proved to be cheaper than the standard design which would have been too expensive becanse of the type of geological formations encountered.

Penstock: a tunnel with a gradient of $1: 1$ driven through the Lias limestone followed by a section through dolomite; thin lining, most of the stresses being taken up by the rock. It was necessary to proceed carefully because of the presence of fissures and clay-filled diaclases, and prestressed underlinings were tested in the dolomite and limestone.
\end{abstract}

suivant : possibilité d'accumuler 200 millions de mètres cubes d'eau entre les cotes $600 \mathrm{~m}$ et $700 \mathrm{~m}$ dans la région la plus arrosée d'Algéria (1 $400 \mathrm{~mm}$ par an en moyenne, dont $800 \mathrm{~mm}$ de ruissellement) à $13 \mathrm{~km}$ de la mer.

Le plan de l'aménagement montre la situation générale des différents ouvrages de cet aménagement. 


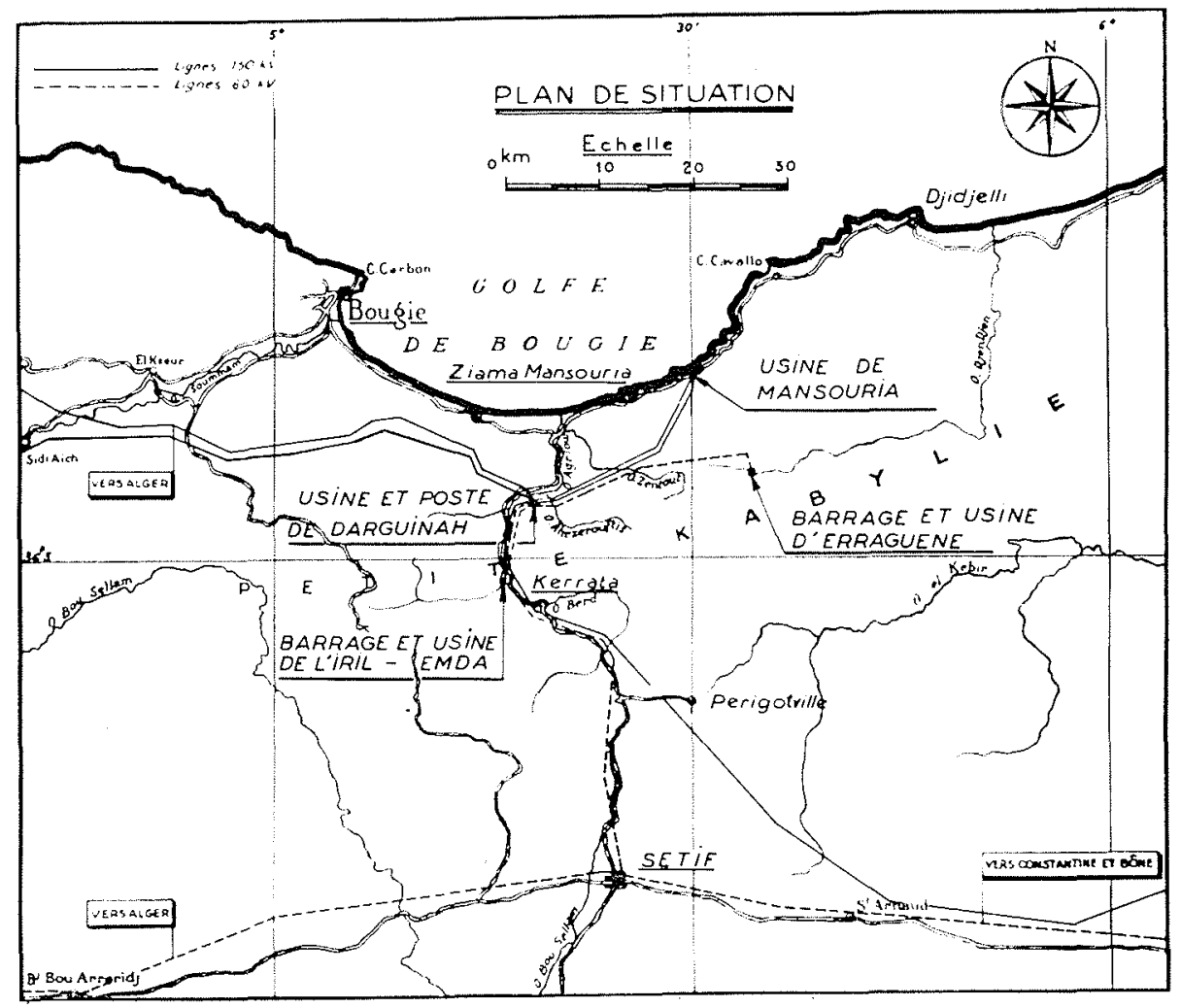

\section{I. - CARACTERISTIQUES GÉNERALES DE L'AMÉNAGEMENT DE L'OUED DJENDJEN SUPÉRIEUR}

L'aménagement de haute chute de l'Oued Djendjen Supérieur comporte d'amont en aval les ouvrages suivants :

1. - Le barrage principal de Merdj Ez Erraguène, implanté dans le verrou aval d'une cuvette schisteuse et qui crée une retenue de $200000000 \mathrm{~m}^{3}$. C'est un ouvrage rectiligne à voûtes multiples (12 de $35 \mathrm{~m}$ de portée d'axe en axe des contreforts), d'une longueur de $530 \mathrm{~m}$ en crête, arasé à la cote $672 \mathrm{~m}$ et d'une hauteur de $86 \mathrm{~m}$.

Les contreforts sont munis à leur pied aval de butées actives équipées de vérins plats système Freyssinet. Ce principe a déjà reçu une application en Algérie au barrage des Beni Bahdel. Il permet de partager la fondation de l'ouvrage en deux parties, séparées par une large bande de terrain faiblement sollicité. La partie amont travaille dans les conditions habituelles; la partie aval, qui comprend les butées actives, permet de mobiliser en arrière de l'ouvrage et en-dessous du niveau général du terrain des réactions importantes peu inclinées sur l'horizontale. Il en résulte une réduction considérable du poids total eau + béton nécessaire à la stabilité de l'ouvrage et, par conséquent, une économie très sensible sur le cube de béton.

Le barrage est également équipé d'ouvrages de dévasement suivant la technique mise au point pour le barrage de l'Iril Emda et dont l'efficacité s'est confirmée de 1954 à ce jour.

2. - L'usine de pied de barrage logée entre deux contreforts équipés d'un groupe vertical à turbine Francis turbinant un débit de $20 \mathrm{~m}^{3} / \mathrm{s}$ et sous $76 \mathrm{~m}$ de chute maximum.

3. - Un barrage-poids secondaire de compensation, créant à l'aval de la restitution de l'usine de pied de barrage une retenue de $300000 \mathrm{~m}^{3}$. Ce barrage a $70 \mathrm{~m}$ environ de longueur et $15 \mathrm{~m}$ de hauteur.

4. - La prise d'eau de l'usine de Mansouria alimentant la galerie d'amenée.

5. - La galerie d'amenée de $3,40 \mathrm{~m}$ de diamètre, de $10455 \mathrm{~m}$ de longueur. Cette galerie 
se raccorde, pour la traversée du col de Djeemâa, à un siphon.

6.-- Ce siphon de $2,60 \mathrm{~m}$ de diamètre et de $1800 \mathrm{~m}$ de longueur est constitué par des tuyaux en béton armé précontraint avec une tôle d'étanchéité posés dans une galerie souterraine revêtue.

7. - A la fin de la galerie du Djebel Breck, de $1260 \mathrm{~m}$ de longueur, qui fait suite au siphon, se trouve la cheminée d'équilibre.

8. - La conduite forcée souterraine de $2,40 \mathrm{~m}$ de diamètre, de $930 \mathrm{~m}$ de longueur et de 0,95 de pente emprunte le flanc nord du Djebel Breck et se raccorde au collecteur à la cote $+6 \mathrm{~m}$.

9. - L'usine de Mansouria, équipée de deux groupes horizontaux de 50 MW avec des turbines Pelton, turbinant chacun $10 \mathrm{~m}^{3} / \mathrm{s}$ sous $590 \mathrm{~m}$ de chute brute.

10. - - La galerie de fuite de l'usine restituant directement à la mer les eaux turbinées.

Le profil en long général indique les principaux ouvrages énumérés ci-dessus (fig. 1).

\section{Position de l'usine dans le complexe algérien}

L'usine de Mansouria, avec ses 100 MW installés, sera essentiellement une usine de pointe et de forte charge. Son utilisation annuelle, en première étape, est de 2400 heures environ. Comme elle sera la plus puissante des usines installée en Algérie au moment de son entrée en service, elle est appelée à jouer le rôle de chef de file.

Elle sera appelée, en outre, à cause de la rapidité de sa mise en service (10 minutes environ) à prendre la charge d'une usine du réseau en défaillance.

Il est également nécessaire qu'elle puisse reprendre rapidement sa charge en cas de déclenchement des deux groupes parce que sa disparition du réseau amènerait de très fortes perturbations et des délestages importants.

Ces considérations ont été à la base des hypothèses retenues pour la conception des ouvrages et du matériel et en particulier des hypothèses d'ouverture et de fermeture pour la cheminée d'équilibre.

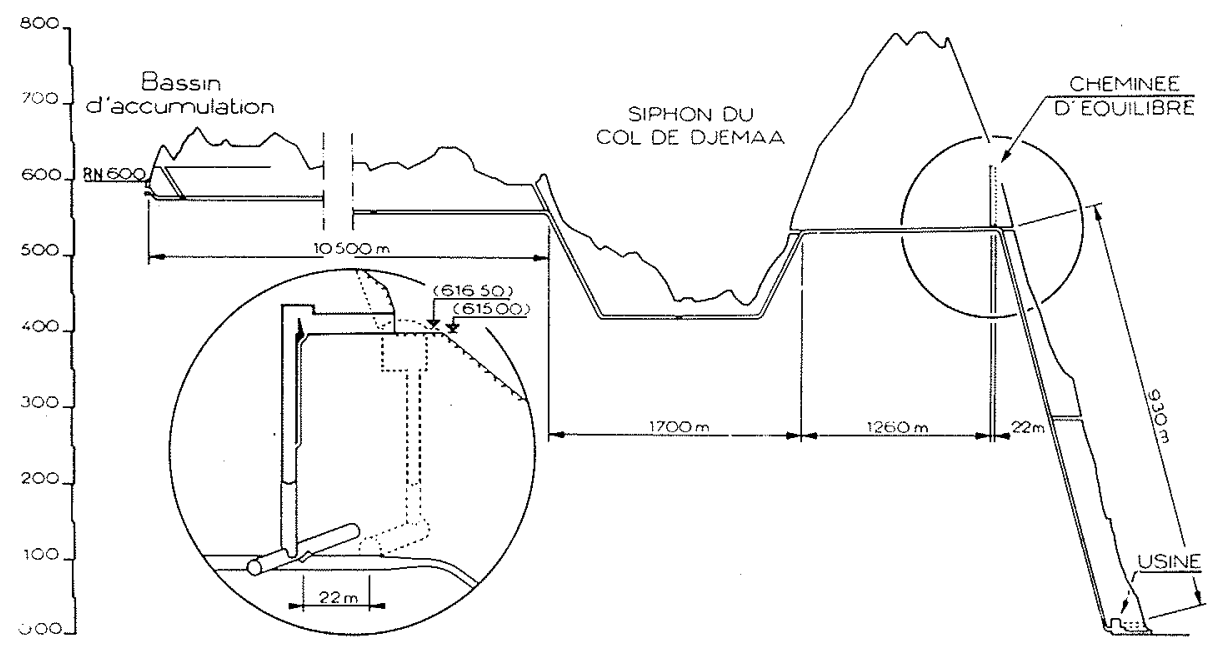

Fic. 1.

Profil en longs genéral

\section{II. - CHEMINÉE D'ÉQUILIBRE}

\section{Hypothèses de départ}

a) Fermeture. - L'hypothèse de fermeture retenue est l'arrêt instantané à pleine charge des deux groupes, le niveau dans le bassin de compensation étant à la cote maximum. $603 \mathrm{~m}$, aucun déversement extérieur n'étant admis et la cote maximum de l'eau dans la cheminée ne devant pas dépasser $621 \mathrm{~m}$ à cause de la pression maximum admissible dans le siphon du col de Djemâa.

b) Ouverture. - Les hypothèses d'ouverture retenues sont les suivantes :

- Passage de la demi-charge $50 \mathrm{MW}$ à la pleine charge $100 \mathrm{MW}$, la cote de la retenue 
dans le bassin de compensation étant au minimum $594 \mathrm{~m}$.

- Reprise instantanée des $3 / 4$ de la charge totale soit $75 \mathrm{MW}$ après un déclenchement brusque des deux groupes, la cote de la retenue étant à $594 \mathrm{~m}$.

- Prise instantanée de la charge totale des deux groupes avec niveau stabilisé dans la cheminée, la cote de la retenue étant au minimum $594 \mathrm{~m}$.

\section{Cheminée prévue au projet Description et fonctionnement}

La cheminée prévue au projet était du type à deux chambres réunies par un puits :

- La chambre supérieure, de $2150 \mathrm{~m}^{3}$ de volume utile, constituée par un cylindre vertical enterré de $14 \mathrm{~m}$ de diamètre, de $14 \mathrm{~m}$ de hauteur, le radier étant à la cote $603 \mathrm{~m}$. Cette chambre, dont le bord supérieur affleurait le terrain naturel était couverte par une coupole en béton armé.

- Le puits de communication, d'un diamètre de $3 \mathrm{~m}$, partait de la cote $603 \mathrm{~m}$ et aboutissait à la cote $544 \mathrm{~m}$ dans la chambre inférieure.

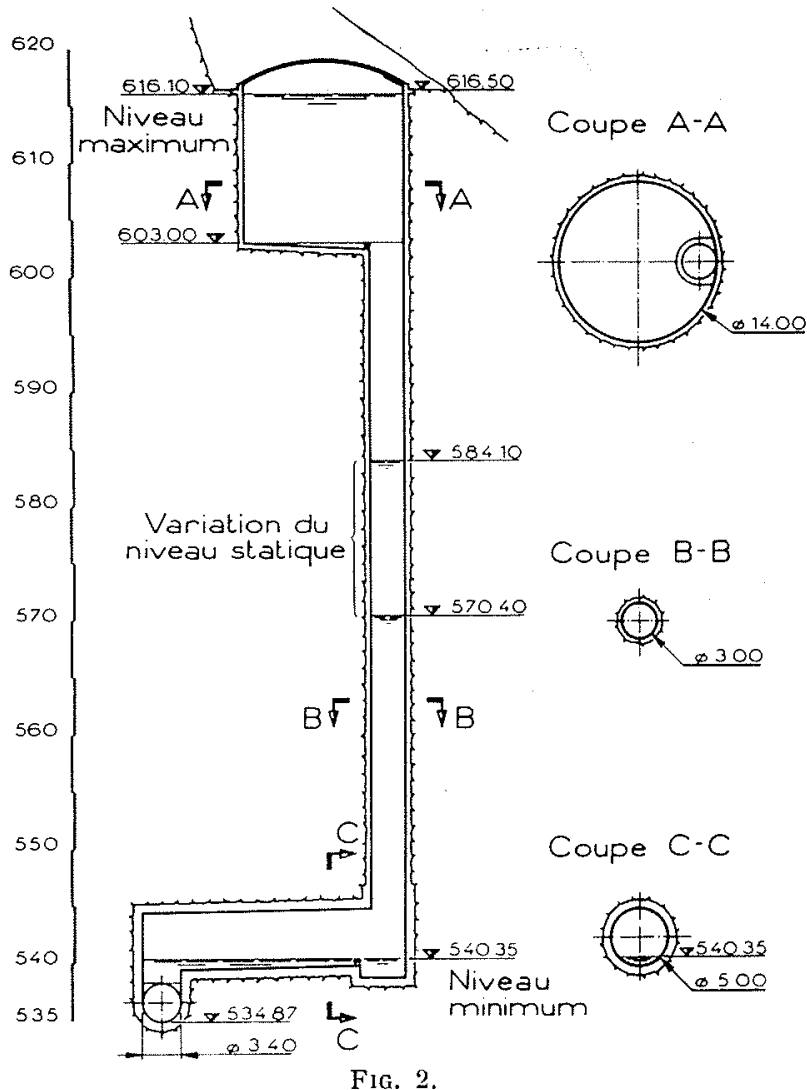

Cheminée d’équilibre. Projet primitif.
-. La chambre inférieure de $1100 \mathrm{~m}^{3}$ de volume utile, constituée par une galerie horizontale cylindrique de $5 \mathrm{~m}$ de diamètre et de $55 \mathrm{~m}$ de longueur. Le radier de cette galerie était au nivean $539 \mathrm{~m}$, soit 0,93 au-dessous de la clef de la galerie d'amenée au point de jonction.

La figure 2 précise les dispositions décrites ci-dessus.

Dans l'hypothèse de fermeture brusque à pleine charge, la montée maximum de l'eau était la cote $616 \mathrm{~m}$ et la cheminée ne déversait pas (le niveau du bassin de compensation ćtant à la cote maximum).

Dans la première hypothèse d'ouverture, passage de la $1 / 2$ charge à la pleine charge dans le cas du niveau minimum dans le bassin de compensation, la descente maximum de l'eau dans la chambre inférieure était de $542,10 \mathrm{~m}$, soit $4 \mathrm{~m}$ environ au-dessus de la clef de la galerie d'amenée.

Dans la seconde hypothèse d'ouverture, reprise des $3 / 4$ de la charge maximum après déclenchement, le niveau minimum de l'eau était de $540 \mathrm{~m}$, ce qui excluait également le risque de dénoyage de la galerie d'amenée.

\section{Exécution}

Lors du percement de la galerie du Djebel Breck prévue dans les calcaires, le terrain rencontré à la cote 534 au droit de l'emplacement prévu par la cheminée d'équilibre se présente sous la forme d'un conglomérat de blocs de calcaires plus ou moins recimentés par de la calcite; ce conglomérat présente des vides importants parfois remplis d'argile.

L'implantation de la cheminée d'équilibro dans un tel terrain présentait des risques; il semble, en effet, que ce conglomérat provienne d'un éboulement ancien dont les blocs ont été ensuite recimentés par des écoulements d'eau chargée et ceci laisse supposer l'existence de cloches ou même de grottes entre le niveau du conglomérat et la surface. Ces accidents pourraient être rencontrés soit sur la hauteur du puits soit dant la chambre supérieure.

Même, si aucun accident n'était rencontré, l'étanchéité des ouvrages posait un problème difficile car il parait dangereux d'admettre des pertes importantes d'eau au-dessus du terrain traversé par la galerie de la conduite forcée.

A une quinzaine de mètres en amont de ces éboulis, une zone de calcaire bleu très sain et très compact fut traversée. Cette zone a une trentaine de mètres de longueur.

L'implantation de la cheminée dans ce calcaire compact fut tout naturellement envisagée. 
Mais au droit de cet emplacement, le terrain naturel en surface est à la cote $645 \mathrm{~m}$ au lieu de $625 \mathrm{~m}$ à l'emplacement primitif. Conserver l'ouvrage prévu nécessitait environ $9000 \mathrm{~m}^{3}$ de déblais rocheux supplémentaires dans un terrain à $45^{\circ}$.

Il n'était pas possible d'autre part :

$1^{\circ}$ De remonter la cote de la chambre supérieure, cette cote étant limitée à 620 par la pression maximum admissible dans le siphon du col de Djemâa;

$2^{\circ}$ D'exécuter la chambre supérieure en souterrain avec sa forme primitive (cylindre vertical de $14 \mathrm{~m}$ de diamètre) étant donnée la faible épaisseur de couverture $(20 \mathrm{~m})$.

Le projet devait être modifié.

Il fallait:

$1^{\circ}$ Caler le plus haut possible la chambre supérieure, c'est-à-dire à $620 \mathrm{~m}$ au maximum;

$2^{\circ}$ Prévoir une chambre souterraine de volume minimum pour pouvoir être exécutée en galerie d'un diamètrè moyen compatible avec la hauteur de couverture.

Notre attention avait été attirée sur l'intérêt des cheminées amortissantes à chambre d'expansion signalé par l'article de MM. BouvarD,

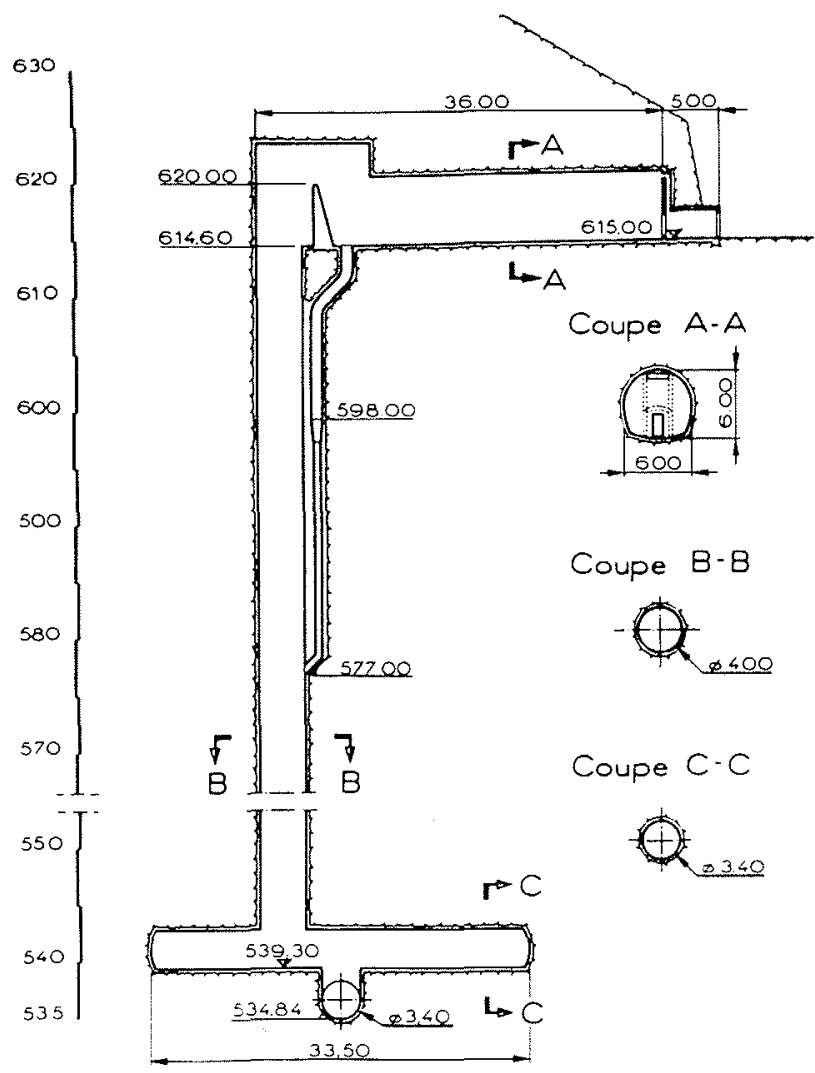

Fig. 3.

Cheminèe d'équilibre. Projet définitif.
Molbert et GÉRARD (Houille Blanche, août/ septembre 1955).

Une cheminée de ce type semblait pouvoir résoudre le problème de façon élégante et économique.

L'Ingénieur-Conseil d'E.G.A., la S.O.G.E.I. Alger, étudia et mit au point la nouvelle cheminée.

Celle-ci comprend :

1. Une chambre supérieure en galerie souterraine en fer à cheval de $6 \mathrm{~m}$ de diamètre et $36 \mathrm{~m}$ de longueur, d'un volume utile de $850 \mathrm{~m}^{3}$ et dont le radier est calé à la cote $614,60 \mathrm{~m}$. Dans cette chambre, sont aménagés :

- un déversoir arasé à la cote $620 \mathrm{~m}$ au débouché du puits de liaison;

- le départ d'un tuyau de $1 \mathrm{~m}$ destiné à vider la chambre supérieure;

- une porte étanche fermant la petite galerie d'accès.

2. Un puits vertical d'un diamètre intérieur de $4 \mathrm{~m}$ et de $72,30 \mathrm{~m}$ de hauteur mettant en communication la chambre supérieure et la chambre inférieure.

3. La chambre inférieure en galerie de $3,40 \mathrm{~m}$

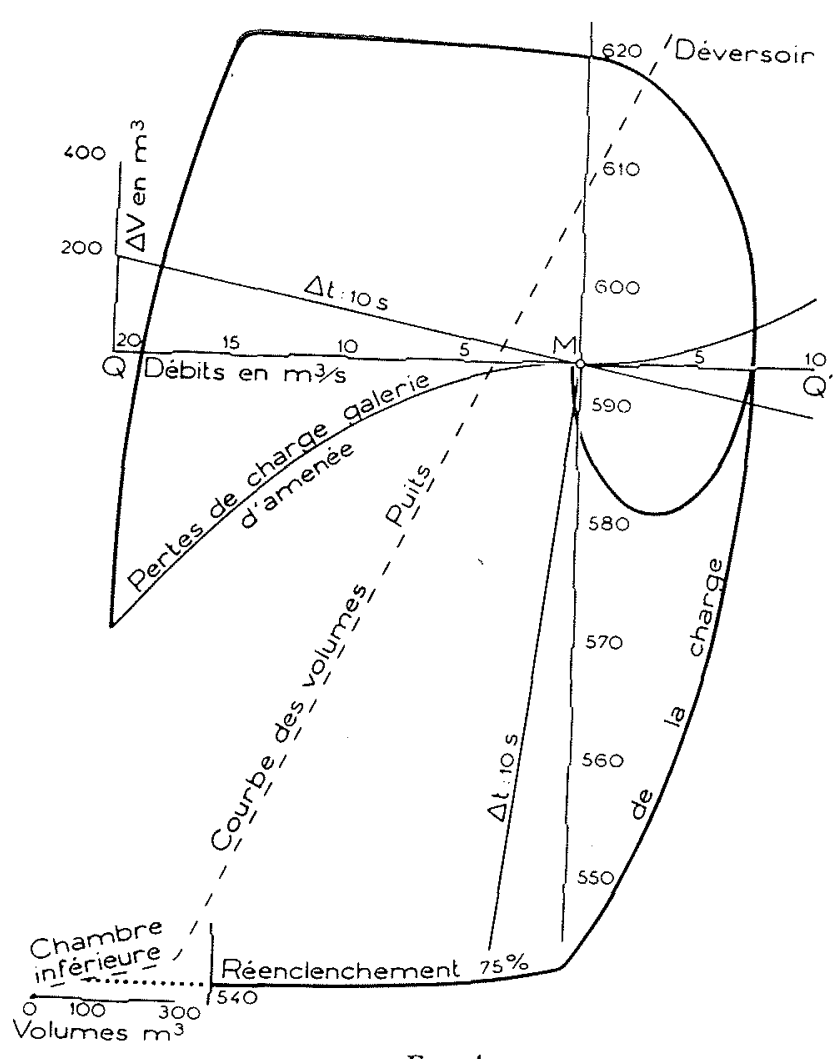

FIG. 4.

Cheminée d'équilibre. Epure. 
de diamètre et de 33 de longueur, d'un volume utile de $300 \mathrm{~m}^{3}$.

4. Le tuyau de vidange de la chambre supérieure, de $1 \mathrm{~m}$ de diamètre, joue le rôle de déphaseur d'énergie. Il part de la cote $614,60 \mathrm{~m}$ et débouche dans le puits à la cote $578 \mathrm{~m}$.

Les dispositions décrites ci-dessus sont explicitées par le croquis $n^{\circ} 3$.

\section{Fonctionnement}

(Dans les mèmes hypotheses que pour le projet primitif.)

\section{Fermeture:}

Lamortissement des oscillations est obtenu à peu près totalement en une seule période, soit 325 secondes, le niveau maximum étant la cote du déversoir, soit $620 \mathrm{~m}$

\section{OUVERTURE :}

1" Passage de la $1 / 2$ charge à la pleine charge. Niveau minimum. (544 m);

2" Reprise de $75 \%$ de la charge de deux groupes après un déclenchement. Le niveau minimum est $540,60 \mathrm{~m}$.

Les épures (fig. 4) montrent qu'aucune sujétion d'exploitation n'est imposée, les manœuvres pouvant se faire à n'importe quel moment.

\section{ECONOMIE :}

Le nouveau projet, évalué au prix du Marché du génie civil, revient à 22 millions au lieu de 27 millions pour le projet primitif.

\section{III. - CONDUITE FORCEE}

\section{Projet du Marché}

La conduite forcée prévue au marché était constituée :

- par une galerie souterraine inclinée de $750 \mathrm{~m}$ environ de longueur terminée par une galerie en palier de $150 \mathrm{~m}$ à la cote $6 \mathrm{~m}$.

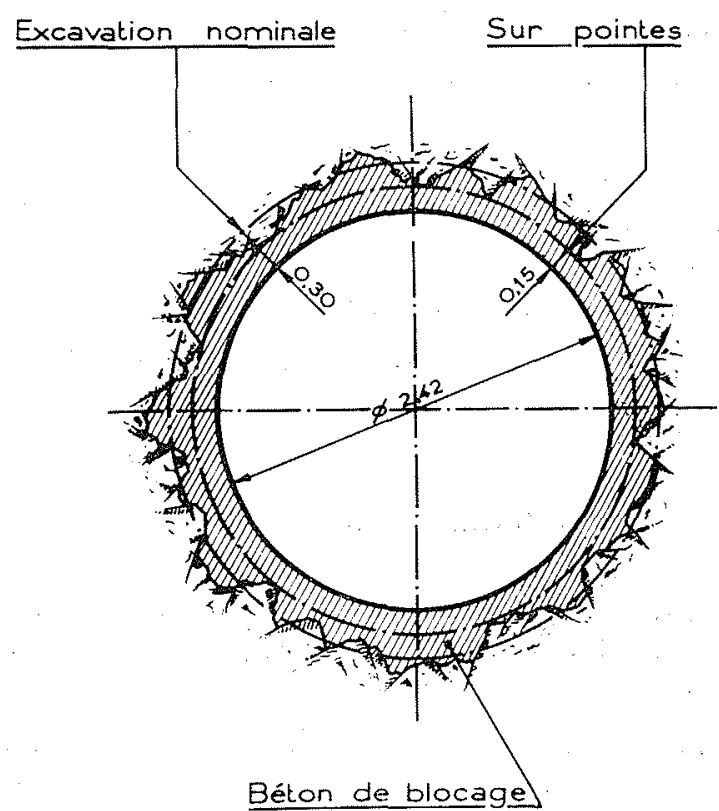

Fic. 5.

Conduite forcée. Coupe transversale.
Le diamitre de l'excavation était de $3 \mathrm{~m}$; - par un blindage, en principe de $8 \mathrm{~mm}$ d'épaisseur en acier doux, nuance chaudière à fort coefficient d'allongement, de $2,42 \mathrm{~m}$ de diamètre.

Un revêtement de béton de $0,30 \mathrm{~m}$ d'épaisseur moyenne remplissait l'espace blindage rocher: (fig. 5)

Dans lés zones où le coefficient d'élasticité du rocher, après tassement initial, n'atteindrait pas la valeur de $150000 \mathrm{~kg} / \mathrm{cm}^{2}$, le blindage mince serait remplacé par du blindage ćpais en acier Confor $\mathrm{D}$ calculé comme travaillant à la limite d'élasticité, soit $34 \mathrm{~kg} / \mathrm{mm}^{2}$, partout où la couverture rocheuse dépasserait $80 \mathrm{~m}$.

Dans les zones à coefficient d'élasticité inférieur à $150000 \mathrm{~kg} / \mathrm{cm}^{2}$ et ò̀ la couverture rocheuse était inférieure à $80 \mathrm{~m}$, la conduite épaisse était calculée comme à l'air libre.

Le projet de blindage mince, inspiré des expériences faites à Rossens, Montpezat, etc., faisait done participer le rocher à la résistance à la pression intérieure.

Les renseignements géologiques indiquaient que la galerie traversait, de la cote $530 \mathrm{~m}$ à la cote $50 \mathrm{~m}$, des calcaires à silex et, au-dessous de la cote $50 \mathrm{~m}$, la dolomie. Ils indiquaient également que le rocher présentait des failles et des diaclases remplies d'argile rouge onctueuse molle. 


\section{Forage de la galerie}

La galerie est à l'heure actuelle entièrement forée.

Elle traverse jusqu'à la cote $50 \mathrm{~m}$ les dolomies et entre 50 et $530 \mathrm{~m}$ les calcaires à silex prévus.

La dolomie, sauf deux accidents localisés, failles ouvertes de 0,10 à $1 \mathrm{~m}$ remplies d'argile molle, paraît très saine et seules des diaclases très fines, sans argile y sont relevées.

Les calcaires présentent des zones fissurées avec des fissures d'épaisseur de 1 à $2 \mathrm{~mm}$ remplies d'argile molle et des zones saines sans argile.

La galerie n'a pas exigé de soutènement, sauf en quelques points très localisés.

La nature des terrains rencontrés est bien celle prévue par les renseignements géologiques et les galeries de reconnarssance.

\section{Etudes complémentaires de la conduite}

L'Entreprise n'avait pas précisé, lors du marché, les essais nécessaires à la détermination des zones devant recevoir soit le blindage mince, soit le blindage épais.

La réalisation d'essais en caverne, qui théoriquement devaient avoir lieu dans chaque zone, s'avéra compliquée et d'un coût élevé.

La présence de zones diversement faillées nécessitait cependant la vérification des dispositions prévues.

La Division Génie Civil du S.P.E.H. de la Direction de l'Equipement d'Electricité de France, fut consultée en janvier 1957 par le maître de l'œuvre qui sollicita son concours pour ces études.

Deux groupes de solution pouvaient être envisagés :

1. Renforcement par surépaisseur du blindage de la conduite;

2. Amélioration de l'assise rocheuse au pourtour du souterrain.

La Division Génie Civil du S.E.P.H. a proposé une solution d'amélioration de l'assise rocheuse par serrage mécanique d'un prérevêtement.

Cette solution avait l'avantage de serrer à la demande du terrain le prérevêtement et de faire travailler le rocher avec un coefficient de déformation plus grand, les tassements initiaux irréversibles étant éliminés par le traitement préalable.

Des essais furent réalisés, l'un dans la dolomie, l'autre dans du calcaire fissuré au niveau $284 \mathrm{~m}$ (fenêtre VII).

Deux variantes, l'une proposée par l'Entreprise, l'autre par E.D.F., furent envisagées :

Dans la première, un prérevêtement découpé en trois voussoirs de $120^{\circ}$, recevait à chaque joint des vérins à pistons exerçant des poussées de 766 t par mètre linéaire de joint (pour la pression maximum de service de $70 \mathrm{~kg} / \mathrm{cm}^{2}$ ).

Ce prérevêtement a un diamètre intérieur de $3 \mathrm{~m}$ pour permettre la pose des viroles de $2,42 \mathrm{~m}$ et le revêtement en béton de 0,30 prévu au marché; son épaisseur variait par paliers (en fonction de la pression de service dans chaque zone) de $0,66 \mathrm{~m}$ à $0,31 \mathrm{~m}$.

La seconde, le prérevêtement a un diamètre intérieur libre de $2,65 \mathrm{~m}$, ce qui laisse pour le blocage du blindage une épaisseur libre de 0,10.

Il est découpé en trois ou quatre voussoirs à $120^{\circ}$ ou $90^{\circ}$ séparés entre eux par des files de vérins plats type Freyssinet noyés dans le prérevêtement lor's de son exécution.

Selon la cote d'application, l'épaisseur de l'anneau ainsi que le nombre de vérins plats sont variables.

Le but de ces essais était d'analyser le comportement du prérevêtement lors de sa mise en compression et de vérifier par des mesures in situ, des caractéristiques telles que :

1. Elasticité du rocher;

2. Valeur des déformations des anneaux;

3. Ouverture des joints des vérins;

4. Résistance et comportement des vérins;

5. Résistance et comportement du prérevêtement;

6. Conditions d'exécution;

7. Permanence de l'effet de précontrainte dans le temps.

\section{Planning des essais}

Il fut décidé d'installer dans le rameau d'essai à la cote 6 quatre anneaux expérimentaux (fig. 6).

Anneau type A. - Solution Entreprise avec deux lignes de vérins à pistons sur les parements et une rotule en ciel.

ANNEAU TyPE B. - Variante solution Entreprise comportant une mise au rond préalable 


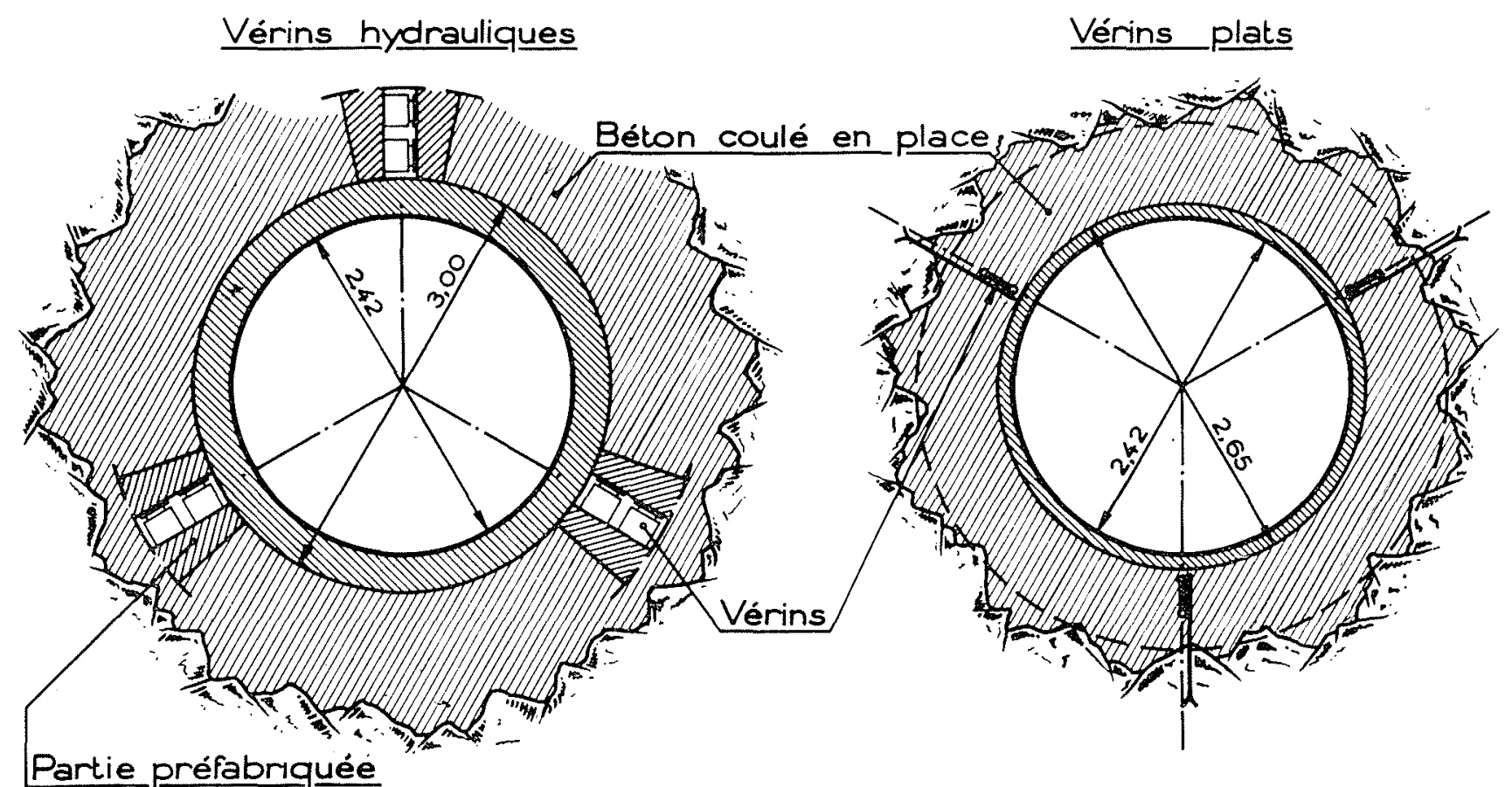

Fig. 6.

Conduite forcée. Anneaux d'essai.

de la galerie par gunitage. Prérevêtement en trois voussoirs équipés de trois files de vérins à $120^{\circ}$ dont une file en radier.

Anneau type C. - Solution Entreprise comportant un prérevêtement bloqué à pleine fouille en trois voussoirs équipés de trois files de vérins à $120^{\circ}$ dont un en ciel.

AnNeaU Type D. - Variante solution E.D.F. analogue à l'anneau $\mathrm{C}$, mais la file de vérins à pistons en ciel est remplacée par des vérins plats.

A la cote 284, deux anneaux ont été expérimentés :

- 1 anneau type $C$;

-- 1 anneau type $E$ solution E.D.F. avec trois files de vérins plats à $120^{\circ}$.

Deux campagnes de mesures ont été effectuées:

- La première, du 13 juin au $1^{\text {er }}$ juillet 1957 avec des contrôles jusqu'en septembre 1957 sur les anneaux $C$ et $D$ du palier et de la cote $6 \mathrm{~m}$ (dolomie);

- La seconde, du $1^{\text {er }}$ au 23 octobre sur les anneaux $\mathrm{C}$ et $\mathrm{E}$ de la cote $+284 \mathrm{~m}$ (calcaires).

\section{Résultats des essais}

Le dépouillement des essais est encore en cours, mais un rapport préliminaire a été édité par la Division Génie Civil du S.P.E.H. de la Direction de l'Equipement d'E.D.F., dont nous donnons les extraits ci-après.

Le coefficient d'élasticité a été calculé d'après les mesures radiales en tenant compte d'un coefficient de forme permettant de reconstituer la déformation relative à un tunnel de même diamètre que l'anneau mais de longueur indéfinie (1).

La pression sur le rocher est calculée par la formule du tube, en supposant l'anneau de béton uniformément comprimé.

Cette hypothèse n'est pas rigoureuse : elle ne tient pas compte en particulier des frottements qui s'exercent entre rocher et béton. Mais le système réel des forces s'exerçant sur le rocher n'en reste pas moins, dans l'ensemble, équivalent à celui calculé.

Le coefficient de déformation $\mathrm{E}$ est déduit de la formule :

$$
\Delta R=(1+u) N / E
$$

$\mathrm{N}$ représentant l'effort normal.

Les résultats sont contenus dans le tableau ci-après :

(1) Un tableau de ces coefficients se trouve page 101 de l'ouvrage Mécanique des Roches, de M. J. TalonRe. 


\begin{tabular}{|c|c|c|c|c|c|c|c|}
\hline $\begin{array}{l}\text { Désisnation } \\
\text { des amneaux }\end{array}$ & $\begin{array}{c}\Delta \mathrm{R} \text { par } \mathrm{kg} / \mathrm{em}^{2} \\
\text { mesuré } \\
\text { en mierons }\end{array}$ & $\begin{array}{l}\text { Coefficient } \\
\text { de forme }\end{array}$ & $\begin{array}{c}\Delta \mathrm{R} \text { par } \mathrm{kg} / \mathrm{cm}^{2} \\
\text { pour un } \\
\text { tunnel indéfini } \\
\text { en microns }\end{array}$ & $\begin{array}{c}\text { Force exercée } \\
\text { par } \\
\text { les vérins } \\
\text { en } t / m\end{array}$ & $\begin{array}{l}\text { Contrainte } \\
\text { dans le } \\
\text { revetement } \\
\text { en } t / \mathrm{m} \\
\text { par } \mathrm{kg} / \mathrm{cm} 2 \\
\text { de pression }\end{array}$ & $\begin{array}{c}\text { Coefficient } \\
1+\mu\end{array}$ & $\begin{array}{c}\text { Coefficient } \\
\text { de } \\
\text { déformation } \\
\text { en } \mathrm{kg} / \mathrm{cm}^{2}\end{array}$ \\
\hline $\mathrm{C}_{6}$ & 0,763 & 0,7615 & 1 & $\begin{array}{l}753,6 \\
753,6\end{array}$ & $\begin{array}{l}1,675 \\
1,675\end{array}$ & $\begin{array}{l}1,20 \\
1,25\end{array}$ & $\begin{array}{l}200.000 \\
210.000\end{array}$ \\
\hline $\mathrm{D}_{6}$ & 0,78 & 0,628 & 1,242 & $\begin{array}{l}759,225 \\
759,225\end{array}$ & $\begin{array}{l}1,687 \\
1,687\end{array}$ & $\begin{array}{l}1,20 \\
1,25\end{array}$ & $\begin{array}{l}163.000 \\
170.0000\end{array}$ \\
\hline$C_{2,2 t}$ & 0,839 & 0,7615 & 1,102 & $\begin{array}{l}753,6 \\
753,6\end{array}$ & $\begin{array}{l}1,675 \\
1,675\end{array}$ & $\begin{array}{l}1,20 \\
1,25\end{array}$ & $\begin{array}{l}183.000 \\
190.000\end{array}$ \\
\hline$E_{2 s t}$ & 4,09 & 0,7615 & 5,37 & $\begin{array}{l}734 \\
734\end{array}$ & $\begin{array}{l}4,9 \\
4,9\end{array}$ & $\begin{array}{l}1,20 \\
1,25\end{array}$ & $\begin{array}{l}110.000 \\
115.000\end{array}$ \\
\hline
\end{tabular}

Les chiffres ci-dessus comprennent la phase de serrage initiale, laquelle correspond toujours à une déformabilité accrue.

L'influence du frottement béton rocher est assez grande et elle a pour effet de réduire les coefficients de déformation calculée. Cette question est à l'étude mais on peut dire que les ordres de grandeur des coefficients seraient de:

$$
\begin{aligned}
& \text { Anneau } \mathrm{C}_{6} \ldots \ldots=190.000 \text { environ } \\
& \text { Anneau } \mathrm{D}_{6} \ldots \ldots=125.000 \\
& \text { Anneau } \mathrm{C}_{284} \ldots=90.000 \\
& \text { Anneau } \mathrm{E}_{284} \ldots=7=
\end{aligned}
$$

avec un coefficient de frottement de l'ordre de 1.

Ces chiffres ne sont qu'une approximation, Ies calculs exigeant pour leur solution, l'introduction d'hypothèses simplificatrices.

Une seconde question encore à l'étude est celle de la permanence de la précontrainte dans le temps.

Les anneaux d'essais ont été maintenus à une pression constante pendant plus de trois mois pour l'anneau $\mathrm{C}_{6}$ et plus de deux mois pour l'anneau $D_{6}$.

Dès à présent, l'examen des graphiques obtenus montre la croissance régulière des déformations suivant une loi logarithmique. D'après le rapport préliminaire E.D.F., les déformations brutales sont dues à des glissements du rocher dont les causes sont connues. Les déformations régulières, par contre, proviendraient du fluage du béton.

La coincidence des courbes des déformations dans le temps obtenues dans les essais avec les courbes de fluage de béton de mềme dosage et de même âge sous des charges très voisines est en effet remarquable. On peut en conclure que le fluage du rocher est bien inférieur à celui du béton et qu'il est même possible, après les tassements initiaux cu'il n'y ait aucun fluage de la masse du rocher sous la charge d'essais.

Pour évaluer l'ordre de grandeur des tassements initiaux en particulier dans le rameau d'essais dans les calcaires à la cote $+284 \mathrm{~m}$, on a effectué après vingt-cing jours de pression maximum constante un cycle de baisse à demipression, un palier de trois jour's à cette valeur et une remontée à la pression maximum.

Il a été constaté :

- La fermeture des joints a été le 1/3 de l'ouverture lors de la montée initiale de la $1 / 2$ pression à la pression maximum. En effet, lors de la montée initiale de la $1 / 2$ pression à la pression totale, l'ouverture totale des trois joints était de 2750 p.Lors de la baisse de la pression totale à la $1 / 2$ pression après vingt-cing jours de pression totale, la fermeture a été de 941 u., soit très approximativement le $1 / 3$;

- Pendant les trois jours de palier à $1 / 2$ pression, l'ouverture des joints n'a varié que de $10 u$ au total.

Lors de la remontée de la $1 / 2$ pression à la pression totale, l'ouverture totale des joints a été de $842 \mu$ soit les 0,90 de la fermeture et de 951 y. après deux jours.

Il semble donc bien que le coefficient de déformation ait été fortement augmenté et qu'à première vue, on puisse dire pour cet annean :

- Les tassements initiaux sont de lordre des $2 / 3$ des déformations tolales;

- Le coefficient de déformation indiqué plus haut, qui est un coefficient basé sur la totalité des déformations, est fortement majoré après la compression du revêtement. 
Bien que ne pouvant encore donner des conclusions définitives sur les résultats de ces essais, il nous a paru que les conclusions provi- soires présentaient un certain intérêt qui valait la peine d'être signalé.

\section{I S C U S I O N}

président: M. FEntr

M. le l'résident précise que le service d'E.D.F. qui a joué le rôle de conseil en ce qui concerne la conduite forcéce désigné par les initiales S.E.P.H., est le Service des Etudes et Projets Hydrauliques de la Direction de liequipement.

M. le Président estime que la communication de M. Méneroun est un bel exemple de l'esprit de recherche srstematique des solutions, à la fois techniquement et economiquement les plus favorables, qui anime nos collèrues d'E.G.A.

M. le Président ouve ensuite la discussion en séparant les deux questions :

1" Chemince d'équilibre,

2" Conduite forcée.

\section{I. - CHEMINÉE D'ÉQUILIBRE}

M. Langlors se demande si lá longueur - telle qu'elle a été prévue - du tuyau d'équilibrage qui descend de la chambre supérieure au puits vertical est nécessaire.

M. Bouvard répond que dans le cas général où l'on emploie le système de la cheminée à chambre d'expansion et tuyau, il est intéressant que le tuyau descende relativement bas pour que la forme de la loi du débit ¿onné par la chambre d'expansion supérieure corresponde a un amortissement aussi rapide que possible. Il est essentiel que le débit donné par la chambre d'expansion ait une allure grossièrement carrée et que, de toutes facons, la vidange de la chambre supérieure ait lieu dans un tomps aussi voisin que possible de celui qui correspond à la demi-oscillation. Comme le débit qui sort de la chambre est donne par la charge sous laquelle il débite, c'est-à-dire en fin de compte, par la longueur du tuyau, on obtient ainsi une relation qui permet de fixer la cote a laquelle il doit descendre.

Dans le cas ò̀ la hauteur de marnage de la retenue est relativement fable, comme sur la chute de Pressy, lo systeme de tuyau n'a pas été employé, mais on lui a préfére un autre type d'ouvrage nettement plus intéressant, dans le cas particulier.

M. Bouvano demande ensuite si les ingénieues qui ont fait le calcul de cheminées d'équilibre ont procédé à une étude systématique pour rechercher quelle était l'influence des variations, telles que le diamètre du tuyau, sa cote et le volume de la chambre d'expansion superienve.

M. Ravo répond que le calcul a été mené pav la méthode graphique de façon a arviver à un nivea final dans la cheminée d'équilibre qui soit la même que to niveau dans le bassin amont, et, d'autre part, à un débil dans la galerie d'adduction pratiquement nul de facon que les oscillations s'amortissent complètement en une période, enfin que, en cas de réenclenchement à $75 \%$ de la charge, on obtienne un niveau dans la cheminéc d'équilibre qui soit sensiblement supérieur au niveau de la salerie dadduction. M. Ravr communiquera cette étude it M. Bouvand.

\section{II. - CONDUITE FORCËE}

Dispositions prises pour assurer la participation du revêtement à la résistance de la conduite forcée.

M. Lasglors souligne l'intèret de l'application ainsi faite du principe de participation du rocher en vue de réduire l'épaisseur d'une conduite torcée en raison des économies qu'elle est susceptible de permettre. Ises essais dont il s'agit ont été étudies puis réalisés sur place par le S.E.P.H. sur la demande d'E.G.A.; mais les resultats ne sont pas encore entièrement dépouillés.

M. LANGlois demande ensuite à MM. Taromie ct Comes qui ont étudié et pour le second, participé, sur place, à leur réalisation, de donner des renseignements complémentaires de ceux que M. MÉneroud pouvait posscider lorsqu'il a rédigé son rapport.

M. 'TaLomre, sans donner de chiffres, souligne l'intérêt plus général de l'homogénéité des déformations mesurées. En calotte, on retrouve les tassements dus au défaut de bourrage si fréquent dans cette partie des revêtements; on trouve aussi des déformations parasites, d'allure erratique, dues au mouvement des véxins par' suite des légers et inévitables défauts de parallélisme de leurs assises. Mais, dans l'ensemble, les courbes de déformées des anneaux restent, dans la plupart des cas, très voisines des cercles, ce qui semble prouver que l'hétérogénéité des roches n'est pas toujours aussi marquée qu'on le suppose. Au Djen-Djen, en résumé, aucune déformation directionnelle privilégiée n'est apparue, et c'est une constatation sur laquelle il paraît utile d'insister.

M. le Président remercie M. Taxobre de ces précisions complémentaires.

M. Bovyard pose les questions suivantes :

- Au point de vue des sous-pressions sur les blindages minces, a-t-on idée de la valeur qu'elles atteindront?

- A-t-on pris des précautions spéciales en rue de faire face à ce risque?

- A-t-on envisagé un puits vèrtical dans le cadre de cet aménagement?

- Peut-on avoir une idée de l'ordre de grandeur de l'économie globale faite par ce procédé, comparé à celui d'une conduite forcée qui encaisserait la pression totale et qui serait Jibre dans une galerie incline?

M. Raud répond qu'au point de vue des sous-pressions, il a été envisagé de mettre quelques ancrages, mais en nombre limité, le terrain - calcaire et dolomie - étant perméable et exempt d'eau en charge.

En ce qui concerne l'économie comparée, celle-ci est largement en faveur du type envisagé, c'est-à-dire conduite mince avec prérevêtement precontraint.

Au sujet du puits, on a finalement envisage un puits incliné.

M. LAzand voudrait savoir si, dans l'expélimontation 
qui a été faite, le parement intérieur en béton était exécuté avant de mettre en pression les voussoirs ou inversement.

M. Ravd répond que les voussoirs ont d'abord été exécutes puis on a placé les vérins et on les a mis en pression. Dans les anneaux d'essai, il n'y avait pas de levétement intéricur.

M. LAZARD demande encore si, lorsque la pression a été maintenue dans les vérins, de juin à septembre, il s'agissait d'une pression hydraulique, ou si l'on avait fait un blocage.

M. Raud explique qu'il s'agissait d'une pression hydraulique qu'on a pu maintenir sans qu'il y ait chute de pression, et qui a toujours été contrôlée soigneusement.

M. LAZARD demande si on a marqué une mesure après le calage pour savoir quel était le fluage du béton su la roche et si on a mesuré la perte dans les calages.

II. RAUD répond négativement à cette dernière question et indique que la mesure du fluage a été faite en maintenant la pression. M. Raud ajoute que les essais ont prouvé la supériorité des vérins plats sur les vérins à piston.

M. 'TaLonRe reprend la question de M. LAZARD et indique dans les anneaux essayés au Djebel Brek, il y a fluage mais, avant tout, il y a fluage des betons tout comme dans tant de structures des travaux publics.

De tels fluages ne devmaient pas poser de problèmes impossibles à résoudre, à supposer même que, dans notre cas, ils puissent poser un problème véritable.

M. le Président remercie M. Raud de son exposé, et le prie de transmettre ses félicitations à M. Ménenoud.

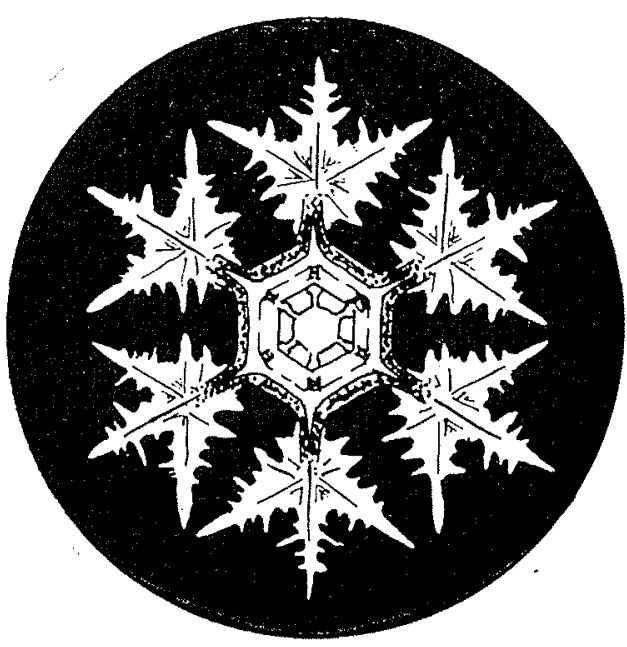

